\title{
Los Docentes de Pregrado de la Universidad Surcolombiana y el uso educativo de las TIC
}

\section{Undergraduate Professors from Universidad Surcolombiana and Educational Use of ICT's}

\author{
Sonia Amparo Salazar Aristizábal \\ Estudiante doctorado en Educación \\ Profesora tiempo completo \\ Universidad Surcolombiana \\ sonia.salazar@usco.edu.co
}

\section{Resumen}

Los docentes en ejercicio hoy en día necesitan estar preparados para ofrecer a sus estudiantes oportunidades de aprendizaje apoyadas en las TIC; al igual que empoderarlos con las ventajas que estas aportan en el proceso de aprendizaje. Es por ello que los gobiernos del mundo tienen dentro de sus políticas el desarrollo de competencias tecnológicas y pedagógicas en sus programas de formación docente.

Esta investigación tuvo como objetivo realizar un diagnóstico sobre el uso de las TIC en la enseñanza de los docentes de pregrado de la Universidad Surcolombiana. La muestra estuvo constituida por 172 docentes y 353 estudiantes pertenecientes a siete facultades y como instrumento de recolección de información se utilizaron cuestionarios cerrados. Los resultados muestran un nivel básico en el uso de las TIC para la enseñanza en los diferentes programas de pregrado.

Palabras clave: Tecnologías de la Información y la comunicación (TIC), profesores universitarios, uso de las TIC.

\section{Abstract}

Abstract

Professor's currently exercising need to be prepared to offer their students learning opportunities supported in ICT's; as well as to empower them with the advantages that these provide in the learning process. That is why governments from around the world have the development of technological and pedagogical competences in their teacher training programs within their policies.

The objective of this research was to carry out a diagnosis on the use of ICT's in teaching of undergraduate professors of Universidad Surcolombiana. The sample was constituted by 172 professors and 353 students belonging to seven faculties and closed questionnaires were used to collect the information. The results show a basic level in the use of ICT's for teaching in the different undergraduate programs.

Key Words: Information and Communication Technologies (ICT's), college professors, use of ICT's.

\section{Introducción}

El conocimiento es en la actualidad un componente central en la conformación de la riqueza y es una condición para el aumento de la productividad y el mejoramiento del nivel de auto reflexión de los ciudadanos. El mundo se ha digitalizado y como consecuencia, se han modificado las relaciones de trabajo, las relaciones sociales y la experiencia humana en general. La era de la información es la nuestra y el Internet, su manifestación más inmediata.

La globalización significa un mundo sin fronteras que induce a que en todos los lugares, todo se parece cada vez más a todo, a medida que la estructura de preferencias del mundo es presionada hacia un punto común homogeneizado. En tales circunstancias, la universidad tiene un doble papel a jugar: de una parte, internacionalizarse para moverse en ese nuevo entorno con pertinencia, y servir de intermediación en el diálogo intercultural, manteniendo un interés central en la identidad nacional. 
El influjo de la globalización ha convertido también la calidad de la educación en un objetivo estratégico para las sociedades: esta nueva situación le plantea a las ciencias, las disciplinas, las profesiones y a la universidad en su conjunto la necesidad de una renovación permanente con el fin de actuar sobre los cambios que se operan en la realidad, esto hace necesario ganar espacios en los ámbitos de poder en la perspectiva de aportar, con base en los saberes producidos en la universidad, a la definición de políticas orientadas al logro de un desarrollo que trascienda la lógica simple del mercado y se ocupe prioritariamente de las condiciones de bienestar y de la calidad de vida de todos.

La comprensión de estas transformaciones exige replantear los paradigmas y crear formas diferentes de relacionarse con los desarrollos científicos, tecnológicos y sus efectos. La Universidad deberá reconstruirse para sobrevivir, desenvolverse en este contexto y brindar respuestas válidas frente a fenómenos complejos como el desempleo, la repercusión social de las nuevas tecnologías, las desigualdades de la globalización, la degradación del medio ambiente, la pérdida de la biodiversidad y los conflictos sociales. La universidad está llamada a crear respuestas nuevas para los cambios profundos llegados con la sociedad del conocimiento. (Rodríguez y Castillo, 2012).

Según Feixas, Marqués y Tomás (1999) la Universidad ante los procesos de cambio científico, tecnológico y social debe prestar atención a la innovación para el cambio, aplicar las TIC y desarrollar proyectos de formación permanente para el personal docente, ya que ellos necesitan estar preparados para ofrecer a sus estudiantes oportunidades de aprendizaje apoyadas en las TIC; al igual que empoderarlos con las ventajas que estas aportan en el proceso de aprendizaje.

El manejo adecuado de las TIC es tan importante que forman parte integral del catálogo de competencias profesionales básicas de un docente, hecho que se ve reflejado en diferentes políticas educativas. En el ámbito internacional la Organización de las Naciones Unidas para la Educación, La Ciencia y la cultura (UNESCO) publicó en el 2008 los Estándares de Competencias en TIC para docentes (ECD-TIC); este documento ofrece orientaciones destinadas a todos los docentes y más concretamente, directrices para planear programas de formación del profesorado y selección de cursos que permitirán prepararlos para desempeñar un papel esencial en la capacitación tecnológica de los estudiantes.

En el ámbito nacional El Ministerio de Educación Nacional en su Plan Nacional Decenal de Educación 2006 - 2016, contempla dentro de sus macroobjetivos la formación permanente de docentes en el uso de las TIC, el fortalecimiento de procesos pedagógicos a través de las TIC, la dotación y el acceso a la infraestructura tecnológica en todas la instituciones educativas y el seguimiento a los proyectos educativos donde se propicie el uso de las TIC.

Así mismo, el Programa Nacional Colombiano de Tecnologías de la Información y las Comunicaciones 2008-2019, uno de cuyos ejes está directamente relacionado con la educación, busca concretamente formar a los docentes en la adquisición de competencia para el uso de las nuevas tecnologías y dotar a las instituciones de herramientas tecnológicas para su aplicación en la educación. Igualmente propone que los programas de Acreditación Institucional de las universidades se transformen de tal manera que permita un mejor conocimiento y aprovechamiento de estas tecnologías por los estudiantes. El uso de estas tecnologías debe ser permanente en el proceso educativo en el aula para todas las materias y en las asignaciones de investigación y desarrollo de trabajos para los alumnos.

En el 2008 el Viceministerio de educación superior publicó el documento Ruta de apropiación de TIC en el Desarrollo Profesional Docente el cual plantea dos procesos dinámicos y permanentes de preparación subjetiva (Sensibilización e Inclusión) que ayudan a enfrentar temores, resistencias o dificultades, o bien, que ayudan a fortalecer, desde lo actitudinal, los aprendizajes mediados por la tecnología. Se espera aportar a la formación de un docente innovador en y desde el uso de las TIC para el fortalecimiento de su área o disciplina de desempeño y el desarrollo permanente de competencias en los estudiantes. Así mismo se formulan dos grandes momentos de preparación cognitiva (Iniciación y Profundización) que ofrecen una línea coherente y escalonada de cualificación personal, profesional en el uso y apropiación de las TIC.

En el 2013 el Ministerio de Educación Nacional publicó el documento Competencias TIC para el desarrollo profesional docente, el cual es una versión actualizada de la ruta de apropiación respondiendo a las demandas del Plan Nacional de Desarrollo 2010-2014 "Prosperidad para todos", que tiene como uno de sus pilares la innovación. Dentro de éste documento la competencia tecnológica se define como la capacidad para seleccionar y utilizar de forma pertinente, responsable y eficiente una variedad de herramientas tecnológicas entendiendo los principios que las rige, la forma de combinarlas y las licencias que las amparan. Esta competencia se desarrolla en tres niveles o momentos que son: exploración, integración e innovación.

En el momento de exploración los profesores empiezan a introducir las TIC en algunas de sus labores y procesos de enseñanza - aprendizaje; en el momento de integración los profesores saben utilizar las TIC para aprender y enseñar de manera no presencial, lo que les permite aprovechar los recursos disponibles en línea, participar en redes y comunidades de práctica; en el momento de innovación los docentes son capaces de adaptar y combinar una diversidad de herramientas tecnológicas para diseñar ambientes de aprendizaje. 
A nivel institucional la Universidad Surcolombiana ha desarrollado jornadas de capacitación relacionadas con el manejo de herramientas tecnológicas ofrecidas a todos los docentes por igual, sin un diagnóstico previo, por tal motivo es necesario conocer el uso que hacen los docentes de la universidad para la enseñanza, con el fin de crear un plan de capacitación docente acorde a las necesidades institucionales. En respuesta a esta situación se plantea el siguiente interrogante.

¿Cuál es el uso de las TIC que hacen los docentes de pregrado de la Universidad Surcolombiana para la enseñanza?

\section{Método}

Para dar respuesta al interrogante anterior se realizó un estudio de carácter cuantitativo con un enfoque exploratorio descriptivo. La recolección de la información se realizó teniendo en cuenta el diseño transeccional descriptivo; los diseños transeccionales recolectan datos en un solo momento, en un tiempo único. Los transeccionales descriptivos tienen como objetivo indagar la incidencia de las modalidades o niveles de una o más variables en una población. (Hernández, 2008). Para la presente investigación se buscó indagar sobre el uso de las TIC para la enseñanza que hacen los profesores de pregrado de la Universidad Surcolombiana sede Neiva. Las variables que se tuvieron en cuenta fueron: el conocimiento de herramientas tecnológicas, el uso de herramientas TIC en los procesos educativos, el diseño de actividades didácticas con herramientas TIC y la actitud hacia las TIC.

La población está constituida por un total de 756 docentes y 8096 estudiantes de la universidad Surcolombiana que forman parte de los programas de pregrado sede Neiva distribuida en 25 programas pertenecientes a siete facultades. Para la selección de la muestra se utilizó una muestra probabilística estratificada proporcional al número de docentes y estudiantes de cada facultad, para determinar el tamaño de la muestra se planteó un margen de confianza del 95\% y 5\% de error estándar. Según Hernández Sampieri en este tipo de muestra la población se divide en segmentos y se selecciona una muestra para cada segmento (pag 180). El total de la muestra corresponde a 172 docentes y 353 estudiantes.

Para recolectar la información se utilizó el método de encuesta, con la técnica de sondeo y como instrumento cuestionario cerrado; este instrumento se aplicó a la muestra seleccionada de docentes y estudiantes de la
Universidad Surcolombiana. Según Giroux y Tremblay (2002, p. 98), el método de la encuesta es un "método que consiste en medir comportamientos, pensamientos o condiciones objetivas de la existencia de los participantes en una investigación a fin de establecer una o varias relaciones de asociación entre un fenómeno y sus determinantes". En este sentido, el fenómeno en estudio de esta investigación, uso de las TIC y sus determinantes el conocimiento, el uso en los procesos educativos, el diseño de actividades didácticas y la actitud hacia las TIC.

\section{Resultados}

Aquí se presentan los resultados más relevantes encontrados dentro de la investigación, los cuales están organizados en: información demográfica, conocimiento de herramientas tecnológicas, el uso de herramientas TIC en los procesos educativos, el diseño de actividades didácticas con herramientas TIC y la actitud hacia las TIC.

Información demográfica. Aquí se presenta información relacionada con el género, edad y nivel educativo y años de experiencia en docencia universitaria de los docentes de la Universidad Surcolombiana. A continuación se presentan los resultados obtenidos en éstos aspectos.

El 64\% de los docentes de la Universidad Surcolombiana son hombres y el $36 \%$ son mujeres.

El 46\% de los docentes de la Universidad Surcolombiana se encuentran entre los 48 y 66 años, el 29\% entre los 37 y 47 años, el $21 \%$ tiene menos de 37 años y un $4 \%$ tiene más de 66 años.

El 59\% de los docentes tienen maestría, el 28\% especialización, el $7 \%$ estudios de doctorado, el $3 \%$ sólo pregrado y el $2 \%$ posdoctorado.

El 27\% de los docentes de la Universidad Surcolombiana tiene una experianica docente de más de 20 años, El 26\% entre 5 y 10 años, el 20\% tiene menos de 5 años y el $12 \%$ entre 16 y 20 años.

Conocimiento de herramientas tecnológicas. Corresponde al conocimiento y uso sobre diferentes herramientas informáticas por parte de los docentes, por tal motivo se indago sobre habilidades instrumentales en el uso de las TIC y el conocimiento sobre normas legales relacionadas con el uso de software educativo y derechos de autor. En la siguiente tabla se muestra los resultados relacionados al nivel de habilidades instrumentales en el uso de las TIC manifestado por parte de los docentes. 
Tabla 1. Habilidades instrumentales en el uso de las TIC.

\begin{tabular}{lcccc}
\hline Habilidades instrumentales / Escala & Excelente & Bueno & Aceptable & Deficiente \\
\hline $\begin{array}{l}\text { Identificación de los componentes básicos } \\
\begin{array}{l}\text { asociados a la tecnología (hardware, software, } \\
\text { redes) }\end{array}\end{array}$ & $30 \%$ & $48 \%$ & $18 \%$ & $4 \%$ \\
\hline $\begin{array}{l}\text { Búsqueda de información por internet. } \\
\text { Edición de documentos en diferentes formatos. }\end{array}$ & $25 \%$ & $50 \%$ & $21 \%$ & $4 \%$ \\
\hline $\begin{array}{l}\text { Elaboración de diapositivas } \\
\text { y /o presentaciones multimedia. }\end{array}$ & $30 \%$ & $48 \%$ & $20 \%$ & $2 \%$ \\
\hline $\begin{array}{l}\text { Uso de hojas de cálculo. } \\
\text { Realización de conversaciones en línea. }\end{array}$ & $12 \%$ & $39 \%$ & $30 \%$ & $19 \%$ \\
\hline $\begin{array}{l}\text { Descarga de documentos en } \\
\text { diferentes formatos de internet. }\end{array}$ & $23 \%$ & $38 \%$ & $24 \%$ & $15 \%$ \\
\hline $\begin{array}{l}\text { Socialización de documentos en } \\
\text { diferentes formatos por internet. }\end{array}$ & $20 \%$ & $37 \%$ & $31 \%$ & $13 \%$ \\
\hline \begin{tabular}{l} 
Realización de trabajos colaborativos en línea. \\
\hline
\end{tabular}
\end{tabular}

Como se puede apreciar en la tabla anterior las habilidades en las que más se destacan los docentes son: identificación de los componentes básicos asociados a la tecnología, búsqueda de información en internet, edición de documentos en diferentes formatos y elaboración de diapositivas; y en la que menos de destacan es en la realización de trabajos colaborativos en línea.

Con relación al conocimiento sobre normas legales relacionadas con el uso de software educativo y derechos de autor, el $54 \%$ de los docentes manifiestan tener conocimiento sobre las implicaciones legales que tiene el uso de licencias para el uso del software comercial en la práctica docente, mientras que un $46 \%$ no tiene conocimiento; El $68 \%$ de los docentes manifiestan tener conocimiento sobre situaciones que implican plagio o fraude en el uso de información digital y un $32 \%$ manifiestan no saber nada al respecto.

Uso de herramientas tecnológicas en los procesos educativos. Se relaciona propiamente con el uso de herramientas y recursos tecnológicos por parte de los docentes en sus actividades pedagógicas por lo tanto los resultados que se presentan están determinado con la frecuencia de uso que hacen los docentes de herramientas y recursos TIC en el proceso educativo. En la tabla 2 se puede apreciar la frecuencia de uso de herramientas tecnológicas y en la tabla 3 la frecuencia de uso de recursos tecnológicos.

Tabla 2. Frecuencia de uso de herramientas tecnológicas.

\begin{tabular}{lcccc}
\hline Herramientas / escala & Siempre & Casi siempre & Algunas veces & Nunca \\
\hline Procesadores de texto & $52 \%$ & $27 \%$ & $18 \%$ & $3 \%$ \\
\hline Presentadores de ideas (diapositivas) & $46 \%$ & $33 \%$ & $19 \%$ & $2 \%$ \\
\hline Hojas de cálculo & $18 \%$ & $20 \%$ & $38 \%$ & $23 \%$ \\
\hline Correo electrónico & $62 \%$ & $23 \%$ & $12 \%$ & $4 \%$ \\
\hline Chat & $23 \%$ & $23 \%$ & $27 \%$ & $26 \%$ \\
\hline Buscadores de información & $45 \%$ & $34 \%$ & $17 \%$ & $4 \%$ \\
\hline Videoconferencias & $8 \%$ & $17 \%$ & $47 \%$ & $27 \%$ \\
\hline Blogs & $8 \%$ & $17 \%$ & $40 \%$ & $35 \%$ \\
\hline Wiki & $5 \%$ & $12 \%$ & $36 \%$ & $47 \%$ \\
\hline Páginas Web & $32 \%$ & $33 \%$ & $23 \%$ & $12 \%$ \\
\hline Bibliotecas virtuales & $22 \%$ & $35 \%$ & $27 \%$ & $16 \%$ \\
\hline Redes sociales & $16 \%$ & $27 \%$ & $33 \%$ & $24 \%$ \\
\hline Foros virtuales & $10 \%$ & $19 \%$ & $41 \%$ & $30 \%$ \\
\hline
\end{tabular}


Como se puede apreciar en la tabla anterior las herramientas que más utilizan los docentes para la enseñanza son: procesadores de texto, presentadores de ideas (diapositivas), correo electrónico y buscadores de información; las que menos utilizan son: foros virtuales, blogs, wikis y videoconferencias.

Tabla 3. Frecuencia de uso de recursos tecnológicos

\begin{tabular}{lcccc}
\hline Recursos tecnológicos / escala & Siempre & Casi siempre & Algunas veces & Nunca \\
& & & & \\
\hline Video beam & $33 \%$ & $38 \%$ & $25 \%$ & $4 \%$ \\
\hline Computador & $47 \%$ & $35 \%$ & $16 \%$ & $2 \%$ \\
\hline Tabletas electrónicas & $8 \%$ & $6 \%$ & $21 \%$ & $65 \%$ \\
\hline Tablero digital & $2 \%$ & $3 \%$ & $10 \%$ & $85 \%$ \\
\hline Sala de informática & $7 \%$ & $9 \%$ & $40 \%$ & $44 \%$ \\
\hline Laboratorio especializado & $7 \%$ & $8 \%$ & $31 \%$ & $54 \%$ \\
\hline
\end{tabular}

Según la tabla anterior los recursos tecnológicos que más utilizan los docentes para la enseñanza son: computador y video beam y los que menos utilizan son los tableros digitales.

Diseño de actividades didácticas con herramientas tecnológicas. Corresponde al uso aplicado en la enseñanza de diferentes herramientas tecnológicas para ello se indago sobre las actividades didácticas con TIC que realizan los docentes y las actividades didácticas que promueven para que realicen los estudiantes. A continuación se presentan los resultados en cada uno de éstos aspectos.

Tabla 4. Actividades didácticas con TIC que realizan los docentes

\begin{tabular}{ll}
\hline Elaboración de presentaciones multimedia (Diapositivas, Videos) & $95 \%$ \\
\hline Diseño de espacios colaborativos (wikis) & $19 \%$ \\
\hline Creación de blogs & $24 \%$ \\
\hline Simulaciones electrónicas de experimentos & $13 \%$ \\
\hline Publicación de trabajos propios a través de internet & $44 \%$ \\
\hline Creación de foros virtuales & $21 \%$ \\
\hline
\end{tabular}

Las actividades con TIC que más realizan los docentes son: elaboración de diapositivas, publicación de trabajos propios a través de internet, mientras que la simulación electrónica de experimentos y el diseño de espacios colaborativos son los menos usados

Tabla 5. Actividades didácticas con TIC que promueven para que realicen los estudiantes en sus trabajos académicos.

\begin{tabular}{ll}
\hline Redacción de trabajos personales y cualquier otro tipo de documento & $90 \%$ \\
\hline Elaboración de presentaciones multimedia & $80 \%$ \\
\hline Realización de búsqueda temática sobre un tópico específico & $75 \%$ \\
\hline Acceso y consulta a bases de datos documentales & $65 \%$ \\
\hline Elaboración de trabajos en forma colaborativa a través de la red & $17 \%$ \\
\hline Elaboración de blog & $13 \%$ \\
\hline Participación en debates e intercambios de mensajes telemáticos (foros virtuales) & $24 \%$ \\
\hline
\end{tabular}

Las actividades que más promueven los docentes que realicen sus estudiantes son: redacción de trabajos personales, elaboración de presentaciones multimedia y búsqueda de información en la web y las actividades menos promovidas son elaboración de blogs y trabajos colaborativos a través de la red.

Actitud hacia el uso de las TIC. En este aspecto se presenta la opinión de los docentes con relación al uso de las TIC en la enseñanza. 
Tabla 6. Actitud de los docentes hacia el uso de las TIC

\begin{tabular}{|c|c|c|c|c|c|}
\hline Afirmaciones / Escala & $\begin{array}{c}\text { Muy } \\
\text { de acuerdo }\end{array}$ & De acuerdo & $\begin{array}{l}\text { Ni de acuerdo } \\
\text { ni en desacuerdo }\end{array}$ & $\begin{array}{c}\text { En } \\
\text { desacuerdo }\end{array}$ & $\begin{array}{c}\text { Muy en } \\
\text { desacuerdo }\end{array}$ \\
\hline $\begin{array}{l}\text { Los docentes deberían utilizar } \\
\text { las TIC para facilitar el aprendizaje } \\
\text { de sus estudiantes. }\end{array}$ & $51 \%$ & $38 \%$ & $9 \%$ & $1 \%$ & $1 \%$ \\
\hline $\begin{array}{l}\text { La utilización de las TIC por parte } \\
\text { de los estudiantes en ciertas } \\
\text { actividades, mejoran el aprendizaje. }\end{array}$ & $41 \%$ & $40 \%$ & $17 \%$ & $1 \%$ & $1 \%$ \\
\hline $\begin{array}{l}\text { Las TIC permiten a los estudiantes } \\
\text { un aprendizaje más individualizado } \\
\text { y adaptado a su propio ritmo. }\end{array}$ & $30 \%$ & $44 \%$ & $19 \%$ & $6 \%$ & $1 \%$ \\
\hline $\begin{array}{l}\text { El docente puede encontrar en las TIC } \\
\text { un buen ayudante para dedicar menos } \\
\text { tiempo a tareas mecánicas. }\end{array}$ & $33 \%$ & $45 \%$ & $15 \%$ & $6 \%$ & $2 \%$ \\
\hline $\begin{array}{l}\text { La Utilización de las TIC por parte de } \\
\text { los docentes se relaciona con una } \\
\text { mayor adecuación de la universidad } \\
\text { a la sociedad en que vivimos. }\end{array}$ & $43 \%$ & $37 \%$ & $16 \%$ & $3 \%$ & $1 \%$ \\
\hline
\end{tabular}

Según los resultados de la tabla anterior se puede apreciar que la mayoría de docentes tiene una actitud positiva hacia el uso de las TIC en el proceso educativo ya que:

El 88\% están de acuerdo en que deberían usar las TIC para facilitar el aprendizaje de sus estudiantes.

El 81\% están de acuerdo en que la utilización de las TIC por parte de los estudiantes en ciertas actividades mejora el aprendizaje.

El 74\% están de acuerdo en que las TIC permiten a los estudiantes un aprendizaje más individualizado y adaptado a su propio ritmo.

El 88\% están de acuerdo en que el docente puede encontrar en las TIC un buen ayudante para dedicar menos tiempo a tareas mecánicas.

El 80\% están de acuerdo en que la Utilización de las TIC por parte de los docentes se relaciona con una mayor adecuación de la universidad a la sociedad en que vivimos.

\section{Discusión}

Para el desarrollo de esta parte se retoman las variables mencionadas anteriormente como son: conocimiento de herramientas tecnológicas, el uso de herramientas TIC en los procesos educativos, el diseño de actividades didácticas con herramientas TIC y la actitud hacia las TIC.

\section{Conocimiento de herramientas tecnológicas.}

Todos los docentes de la Universidad Surcolombiana tienen conocimiento de herramientas tecnológicas como las ofimáticas (editores de texto, presentación de ideas, hojas de cálculo) y búsqueda de información en la web; sólo unos pocos manifiestan tener conocimiento de las herramientas de la Web 2.0. Por otro lado más de la mitad de los docentes tienen conocimiento de las normas legales del uso de software y propiedad intelectual.

El desconocimiento de las diferentes herramientas de la Web 2.0 es preocupante debido al gran auge que ha tenido y las ventajas infinitas que se pueden sacar de estas en el proceso enseñanza aprendizaje. 
Uso de herramientas TIC en los procesos educativos. Las herramientas que más utilizan los docentes para la enseñanza son: procesadores de texto, presentadores de ideas (diapositivas), correo electrónico y buscadores de información y las que menos utilizan son: foros virtuales, blogs, wikis y videoconferencias.

El uso de las diferentes herramientas por parte de los docentes se corresponde con el conocimiento y habilidades que poseen con relación estas.

Los recursos tecnológicos que más utilizan los docentes para la enseñanza son: computador y video beam y los que menos utilizan son los tableros digitales y laboratorios especializados. El motivo por el cual los docentes no utilizan casi estos recursos tecnológicos se debe a la falta de infraestructura y capacitación en el manejo de éstas.

\section{Diseño de actividades didácticas con herramientas TIC.}

Las actividades con TIC que más realizan los docentes son: elaboración de diapositivas, publicación de trabajos propios a través de internet, mientras que los diseños de actividades colaborativas son los menos usados.

Las actividades que más promueven los docentes para que realicen sus estudiantes son: redacción de trabajos personales, elaboración de presentaciones multimedia y búsqueda de información en la web y la actividad menos promovida es la elaboración de blogs.

Tanto las actividades que realizan los docentes en la enseñanza como las actividades que promueven se corresponden con el conocimiento y habilidad en el uso de las mismas que cada uno tiene.

\section{Actitud de los docentes hacia el uso de las TIC.}

Los docentes consideran que el uso de las TIC es importante ya que estas facilitan y mejoran el aprendizaje de los estudiantes y permiten que aprendan a su propio ritmo.

Creen que la incorporación de éstas en la universidad, constituyen un factor de mejora en la calidad de la docencia y deben usarse por todos los docentes en las diferentes asignaturas.

Este hecho es un factor determinante en el uso de las TIC pues todos los docentes son conscientes de la importancia de éstas y la necesidad que tienen de capacitarse.

\section{Conclusiones y Recomendaciones}

Los docentes de la universidad Surcolombiana son conscientes de la importancia de usar las TIC en la enseñanza por lo que requieren mayor capacitación en el uso de las mismas.
A pesar de las políticas educativas que existen con relación al conocimiento y uso de las TIC en la enseñanza, la Universidad Surcolombiana está muy lejos de su meta ya que no cuenta con una infraestructura adecuada, pues no hay salas multimedia suficientes y la conectividad es bastante deficiente, la red se cae con frecuencia.

Debido a la falta de infraestructura tecnológica el uso de las TIC por parte de los docentes en la Universidad Surcolombiana se limita al uso de computador y video beam, recursos tecnológicos que los docentes llevan a sus clases.

De acuerdo a los enfoques propuestos por la UNESCO en los estándares de competencia en TIC para docentes podemos apreciar que lo que más usan los profesores de la Universidad Surcolombiana para la enseñanza son las herramientas ofimáticas y los buscadores de información y lo menos usado corresponde con las herramientas de la Web 2.0 hecho que se deben al desconocimiento de éstas.

Es necesario que la administración se preocupe por mejorar la infraestructura tecnológica y realice mantenimiento constante a los equipos y la red que posee.

Es importante que se haga un plan de capacitación docente en TIC teniendo en cuenta las diferentes herramientas gratuitas que posee la Web 2.0 y que no se limite sólo al uso de la Plataforma Moodle.

Es indispensable hacer una revisión a cada uno de los laboratorios y salas de informática para verificar el cumplimiento de los requisitos mínimos de cada uno de éstos espacios, igualmente es necesario dotarlos con equipos de última tecnología.

\section{Referencias bibliográficas}

Briceño, J. J., González, M. F., y Mosquera, A. J. (2012) "Estrategia de formación de docentes y estándares de competencia en TIC propuesta Colombia". Bogotá.

Ministerio de Comunicaciones (2008). Plan Nacional de Tecnologías de la Información y las Comunicaciones. Bogotá.

Ministerio de Educación de Colombia (2006). Plan Nacional Decenal de Educación 2006-2016: Pacto Social por la Educación. Bogotá.

Ministerio de Educación de Colombia (2013). Competencias TIC para el desarrollo profesional docente. Bogotá.

Duderstadt, J. J., Atkins, D. E, y Van Houweling, D (2002) "Higher Education in the Digital Age: Technology Issues and Strategies for American Colleges and Universities". American Council on Education and Praeger Publishers. Westport, CT.

Giroux, S. y Tremblay, G. (2002). Metodología de las ciencias humanas. México: Fondo de Cultura Económica.

Hernández S. Fernández C., y Baptista, Pilar (2010). Metodología de la investigación. México: McGraw Hill, 2010.

Organización de las Naciones Unidas para la Educación, la Ciencia y la Cultura -UNESCO (2008). Estándares de Competencia en TIC para Docentes (ECD-TIC). Londres.

Pablos. J, Area. M, Valverde. J, Correa. J (2010). Políticas Educativas y Buenas Prácticas con TIC, Barcelona, España. Grao. 2010

Rodriguez. J, y Castillo, Nydia (2012). La Educación y su Futuro. Congreso: Panamá. Virtual Educa

Tapscott, D (2010). Grown up Digital. McGraw Hill. New York. 